\title{
Gas in the orbit associated with orbital cellulitis and paranasal sinusitis
}

\author{
*D. SEVEL, *B. TOBIAS, †S. L. SELLARS, AND $\ddagger$ A. FORDER \\ From the Departments of *Ophthalmology, †Otolaryngology, and $\ddagger$ Bacteriology, Groote Schuur Hos- \\ pital, and the University of Cape Town, South Africa
}

The association of gas in the orbit with paranasal sinusitis and orbital cellulitis has not hitherto been recorded.

\section{Case report}

A coloured male, aged 16 years, whose occupation was a metal grinder, was first seen in the Eye Out-Patients' Department at Groote Schuur Hospital on July ı6, I97 I. He gave a 3-day history of a sudden onset of pain in the left eye followed by swelling of the lids and proptosis. He could not recall sustaining trauma to the left eye nor could he recall being injured by a foreign body entering the eye. About the same time he developed a cough productive of purulent sputum. He was referred to the Department of Ophthalmology by a medical practitioner who had treated the patient with parenteral penicillin for 2 days.

\section{General examination}

He was toxic, somewhat confused, and pyrexial. The left eye was grossly proptosed and was surrounded by tensely swollen lids (Fig. I). The eye could not be reduced into the orbit and any attempt to do so caused discomfort. The conjunctiva was congested, haemorrhagic, necrotic, and friable (Fig. 2). Exposure keratitis was present as evidenced by a dry ulcerated cornea. Corneal sensation was absent. The pupil was fixed and dilated and did not react to direct or consensual light stimulation. Extraocular movements were absent and the eye could not be moved passively. There was no perception of light. The right eye was normal in all respects and the visual acuity was $6 / 6$.

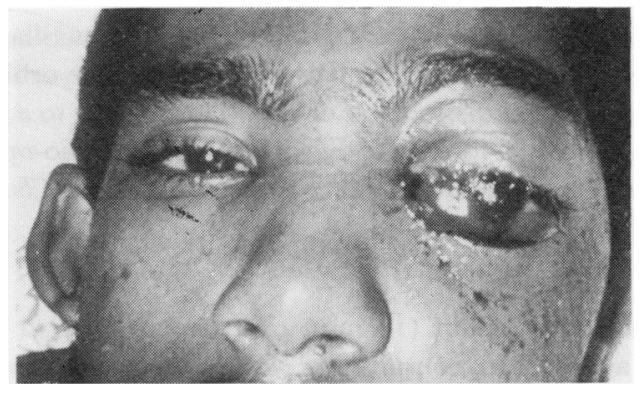

FIG. I Left eye grossly proptosed and surrounded by tensely swollen lids

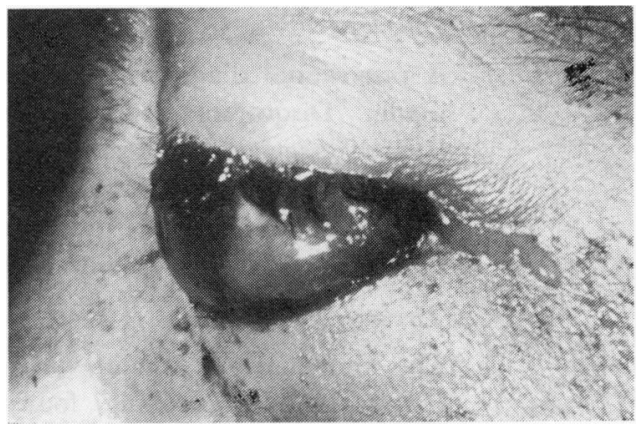

FIG. 2 Conjunctiva congested, chemotic, and haemorrhagic 
Systemic examination

The respiratory rate was 32 per min., the temperature $38 \cdot 2^{\circ} \mathrm{C}$., the pulse 80 per min., and the blood pressure 150/1 10. The trachea was deviated to the left. There was poor air entry from to lung fields, where diffuse coarse rhonchi were heard. Chest movement was poor because of kyphoscoliosis. The patient was coughing up purulent sputum.

Radiology

The chest showed increased vascular markings at the base, and the cervical vertebrae showed fusion of the $3^{\text {rd }}$ and $4^{\text {th }}$ cervical bodies. Other features of the Klippel-Feil Syndrome were a short neck with a low hairline. $X$-ray of the left orbit showed considerable soft tissue swelling and a welldefined bubble of gas could be seen in the orbital tissue (Fig. 3). No radio-opaque foreign bodies were observed and there were no fractures of the orbital walls. No osteitis or erosion of the bony wall of the orbit were noted. The ethmoidal sinus and left maxillary antrum were opaque (Fig. 3).

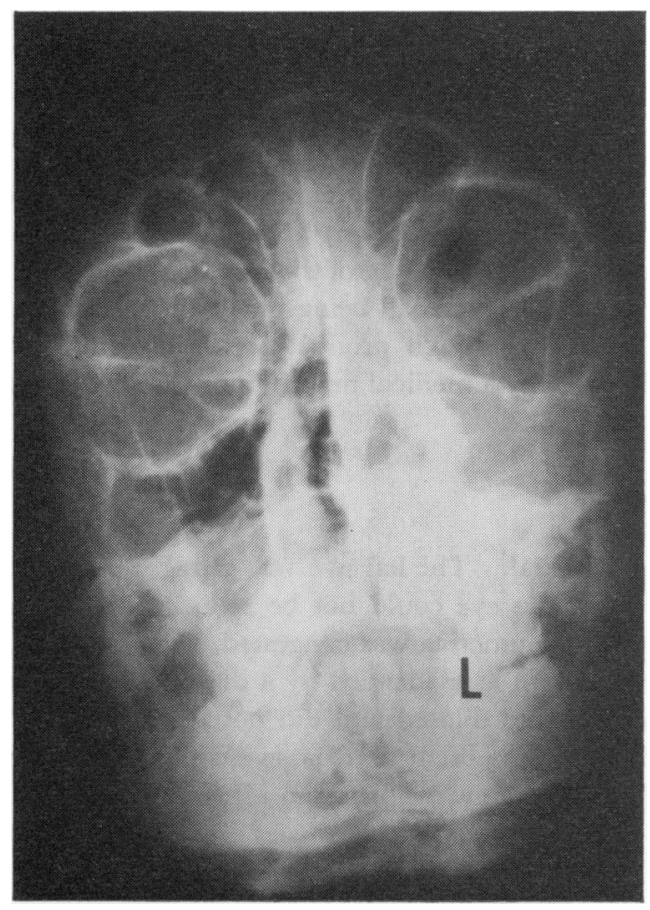

FIG. $3 X$-ray of left orbit, showing a welldefined bubble of gas in the orbital tissue. The ethmoidal sinus and left maxillary antrum are opaque

\section{Diagnosis}

A provisional diagnosis of orbital cellulitis associated with ethmoiditis and maxillary antritis was made. Disquieting features were the severe toxic state of the patient and gas in the orbit. The latter is rarely associated with sinusitis and a provisional diagnosis of orbital cellulitis due to a gas-producing organism was contemplated. The possibility of a foreign body which was not radio-opaque was also considered. In view of previous experience (Bristow, Kassar, and Sevel, 1971), Clostridium welchii was suspected as the offending organism.

\section{Therapy}

The immediate treatment was intravenous penicillin ( $10 \mathrm{~m} . \mathrm{u}$. over a period of $12 \mathrm{hrs}$ ) and oral chloramphenicol $500 \mathrm{mg}$. four times a day. In consultation with the general surgeons, it was decided not to give the patient anti-gas gangrene serum because of its doubtful efficacy and because of the danger of anaphylactic shock. Furthermore, at this stage, the diagnosis of gas gangrene of the orbit had not been confirmed. 


\section{Operation}

The patient was taken to the operating theatre at I. I 5 a.m. on July 17, 197 I.

The general anaesthesia was a particularly hazardous undertaking because of the purulent bronchitis. The trachea was deviated markedly to the left side as part of the Klippel-Feil syndrome. The major problem was to achieve and maintain an air-way for anaesthesia. It was decided that the only safe method was to intubate the patient under local anaesthesia (Lignocaine 4 per cent.) with the patient awake. It was not possible to view the vocal cords because of a considerable collection of purulent secretion above the epiglottis. Immediately the endotracheal tube was in position $25 \mathrm{mg}$. Thiopentone sodium was given intravenously. Anaesthesia was maintained on a semi-closed circuit of the Boyle's machine with oxygen, nitrous oxide, and methoxyfluorane. During the entire procedure the systolic pressure was maintained at $100 \mathrm{~mm}$. Hg. As hyperbaric oxygen was to follow the surgery, myringotomy tubes were inserted into the eardrums before the major surgery. Under general anaesthesia it was noted that the eye was proptosed beyond the equator and the cornea was xerotic and opaque. The eye felt soft digitally but was fixed and could not be moved passively. The bulbar conjunctiva was friable and necrotic and peeled off the eye when an attempt was made to lift it with forceps.

The site of maximum skin swelling and oedema was supra-temporal. A 3-cm. skin incision was made at this location and extended deeply as far as the supraorbital ridge. At this stage foulsmelling sanguineous thick creamy pus bubbled out of the wound. As the little finger was inserted into the wound, the eye ruptured and an expulsive haemorrhage occured through the corneal dehiscence. Because of the poor state of the patient (marked purulent secretions welling up through the endotracheal tube) it was decided to remove the eye remnants and as much of the necrotic orbital tissue as possible. Most of the necrotic soft tissue was located on the lateral side of the orbit. A further large pocket of pus was found at the apex of the orbit. No orbital foreign bodies were noted or palpated. The medial wall of the orbit was intact, the periosteum was not interrupted, and there was no necrosis or pus in relation to the medial side of the orbit. No overt connection between the orbit and the ethmoid or sphenoidal air cells was noted. The bleeding was stemmed by direct pressure with hot gauze ribbons. The skin wounds were loosely apposed by four 6-o black silk sutures. A corrugated tube was fixed in position in the orbit to allow for further drainage. The pus was immediately sent for bacteriological investigation. A Gram-stained smear of the pus revealed numerous pus cells, Gram-positive cocci, pleomorphic Gram-positive bacilli morphologically resembling diphtheroids and Gram-negative bacilli.

At 3.30 a.m. that morning, the patient was sent for hyperbaric oxygen treatment. The first treatment was started approximately an hour after the operation. He received 2 hours' treatment in a single-patient hyperbaric oxygen chamber (high pressure oxygen chamber Mark 3) filled and ventilated with 100 per cent. oxygen with a pressure of 3 atmospheres (approximately 30 lb. per sq. in.). He was compressed without any prior sedation or analgesia. Approximately I hour after starting the patient became restless and complained of severe orbital pain. He was then decompressed, 10 mg. Omnopon and $25 \mathrm{mg}$. Sparine were administered intramuscularly, and he was then returned to the oxygen chamber and re-compressed to complete his 2-hour treatment. He was then sent back to the ward.

He returned approximately 4 hours later for the second 2-hour treatment. Later the same day a left maxillary sinus washout was performed and this produced a moderate amount of sanguineous purulent discharge. Maxillary sinus lavage via an indwelling catheter was continued for a further 4 days until there was no further discharge. The pyrexia settled to within normal limits over the first 48 hours and as there was no drainage from the orbital cavity, the drain was removed.

\section{BAGTERIOLOGIGAL INVESTIGATION}

The pus was cultured both aerobically and anaerobically. No growth was obtained from the solid media inoculated, but subsequent isolations were made from the liquid media (i.e. serum broth and the cooked meat medium). A Gram-stained smear of the serum 
broth revealed Gram-positive cocci (which appeared degenerate). An alpha-haemolytic $\frac{0}{5}$ streptococcus was cultured from the serum broth and the cooked meat medium. No $\frac{0}{3}$ other organisms were isolated from these two liquid media, but a Gram-stained smear $\stackrel{0}{.}$ of the cooked meat medium revealed fusiform Gram-negative bacilli and Gram-positive $\underset{\Rightarrow}{\vec{B}}$ diplococci. At no time were Clostridia isolated and in none of the Gram-stained smears (including the Gram-stained smear made from the original pus) were any organisms seen which might conceivably have been clostridial species.

\section{HISTOLOGY}

The orbital tissue and extraocular muscles submitted for histology showed marked necrosis. Secondary necrotizing arteritis was observed. Sheets of polymorphonuclear leucocytes and $\vec{\omega}$ bacteria were present. No fungi were noted with periodic acid-Schiff stain. The eye was collapsed and contained only blood clot. Perforation had occurred at the site of the corneal thinning. The remaining cornea showed absence of corneal epithelium and Bowman's membrane (probably denuded during the operation). The scleral shell and optic nerve stump showed no abnormality.

Result

The postoperative course was uneventful and the patient was discharged on July 3 I, 1971, when there was minimal discharge from the orbit. Routine follow-up has shown a satisfactory orbit with healing, apart from minimal discharge, and the sinus infection has resolved without further complication and without the need for further surgery.

\section{Discussion}

Acute paranasal sinus infection may be associated with a "sympathetic" reactive oedema of the orbit, orbital cellulitis, and subperiosteal abscess. Optic neuritis, cavernous sinus thrombosis, and even blindness have also been described (Jarrett and Gutman, 1969). The clinical progression from an acute paranasal sinus infection to intraorbital cellulitis may be rapid (as occurs in children), but this rarely progresses to abscess formation. There are no lymphatics in the orbital cavity and therefore the mode of spread from the paranasal sinus to the orbit occurs either directly through the intervening bone or via the venous pathways that pass from the orbit medially to the nasal veins through the ethmoidal cells. These veins contain no valves (Duke-Elder, I96I). The bony confines of the orbit may be dehiscent, thus facilitating rapid spread of infection into the orbit from any of the paranasal sinuses (Shaw, 1964). The presence of gas in the orbit without direct connection with the sinuses or with dehiscence of the orbital wall implicates either spread of a gas-producing organism from the sinus into the orbit or a septicaemia with localization in the orbit. The latter is most unlikely in this case as there was no further evidence of septicaemia. It is clear that the foul-smelling pus and the gas production could not be due to the alpha-haemolytic streptococci which were isolated. Furthermore, a clostridial species could not be implicated since there was no evidence of this organism in the smears or on culture. Extremely foul-smelling pus would tend to point to an anaerobic organism and the possible clue to the cause of the infection in this patient might be the fusiform Gram-negative bacilli noted in the smear made from the cooked meat medium. The genus Fusiformis comprises a variety of anaerobic Gram-negative non-sporing bacilli, all obligate parasites and some pathogenic for man. The group of organisms known as Bacteroides belongs to this genus. The species is characteristically pleomorphic and 
particularly malodorous on culture, and certain species produce gas. In many instances, isolation is difficult and this is especially so if the specimen, for example an ordinary swab, is exposed to the air, This is due to the extreme oxygen sensitivity of many strains of fusiform bacilli. Another frequent difficulty is overgrowth of the fusiform organisms by accompanying bacteria. Fusiform organisms form part of the normal flora of the mouth, the intestinal tract of man, and the female genital tract. They may be present, often in association with other organisms and especially anaerobic cocci, in such pyogenic infections as brain abscesses, peritonitis, endometritis, pyometria, otitis media, stomatitis, sinusitis, and septicaemia (Trevor Willis, 1960). Another reason for the difficulty in isolating this organism may have been the heavy antibiotic cover given to this patient before surgery.

The most important clue in this particular patient is the extremely foul-smelling pus. The aerobic gas-producing enterobacteria (Proteus species, Klebsiella species, and Escherichia coli) do not produce an unpleasant smell. In this patient other anaerobic organisms which could have been responsible for a foul odour are the anaerobic cocci. These anaerobic cocci have been isolated from cases of salpingitis, pulmonary gangrene, appendicitis, broncho-pneumonia, tonsillitis, and acute bacterial endocarditis after septic abortion. These organisms can also produce gas.

\section{Summary}

A patient with gas in the orbit associated with paranasal sinusitis and orbital cellulitis is described. The treatment was excision of the eye and the necrotic tissue, antibiotic cover, and hyperbaric oxygen. The possible causative organism is considered.

This investigation was financed by the Gratitude Fund, the Goosen Fund, and the Department of Ophthalmology Research Fund.

\section{References}

BRISTOW, J. H., KASSAR, B., and SEVEL, D. (197I) Brit. F. Ophthal., 55, I 39

JARrett, W. H., and gutman, F. A. (1969) Arch. Ophthal. (Chicago), 81, 683

DUKE-ELDER, s. (196I) "System of Ophthalmology", vol. 2, p. 479. Kimpton, London

SHAW, H. J. (1964) Proc. roy. Soc. Med., 57, 742

Willis, A. TREVOR (1960) "Anaerobic Bacteriology in Clinical Medicine", p. 87. Butterworth,

London 